\title{
GOBERNANZA INTERCULTURAL
}

\author{
José G. Vargas-Hernández ${ }^{1}$
}

Resumen. El objetivo de este trabajo es abonar con las reflexiones y el análisis al debate sobre la gobernanza intercultural como la mejor alternativa propuesta de los modelos y sistemas de gobernanza existente para todos los niveles institucionales y organizacionales. Se cuestionan fuertemente los modelos monoculturales, multiculturales y de diversidad cultural existentes y su viabilidad en la complejidad de los procesos de globalización. El método empleado es el analítico. El enfoque teórico metodológico se fundamenta en la teoría de contingencias bajo los supuestos de que las situaciones y contextos específicos en relación a las personas y grupos de interés varían en condiciones y requieren por lo tanto diferentes diseños y estructuración de gobernanza intercultural de espacios de diálogo, formas de participación ciudadana, desarrollo y formación de competencias interculturales y diseño del modelo de gestión de la gobernanza intercultural. Para concluir, se presentan las tendencias y principales retos que se plantean con la implementación del modelo de gobernanza intercultural.

Palabras clave: Gobernanza intercultural, diálogo intercultural, participación ciudadana, competencias interculturales, gestión de gobernanza intercultural.

Abstract. The aim of this work is paid with reflections and analysis to the debate on intercultural governance as the best alternative proposed of models and systems of governance for all existing institutional and organizational levels. Monocultural, multicultural and cultural diversity existing models and their viability in the complexity of the processes of globalization is strongly questioned. The method employed is analytical. The methodological approach is based on the theory of contingencies under the assumption that the situations and contexts in relation to individuals and interest groups vary in terms and therefore require different designs and governance structure of intercultural dialogue spaces, forms of citizen participation, development and training of intercultural skills and design management model of intercultural governance. To conclude, the main trends and challenges in the implementation of intercultural governance model are presented. Keywords: intercultural governance, intercultural dialogue, citizen participation, intercultural skills, intercultural management governance.

\footnotetext{
${ }^{1}$ M.B.A.; PhD. Adscrito al Centro Universitario de Ciencias Económico Administrativas. Universidad de Guadalajara, Pertenece al SNI Nivel 1. Datos de contacto Periférico Norte 799 Edif. G201-7. Núcleo Universitario Los Belenes Zapopan, Jalisco, 45100, México Telefax: 523337703340 ext. 256585. jvargas2006@gmail.com,jgvh0811@yahoo.com,josevargas@cucea.udg.mx
} 


\section{Introducción}

Este trabajo busca contribuir con las reflexiones al debate sobre la gobernanza intercultural. Las nuevas expresiones del pensamiento universal sobre las preocupaciones del desarrollo de los pueblos, conducen naturalmente a determinados acercamientos acerca de la gobernanza intercultural global. En la creciente complejidad de los procesos de globalización adquiere relevancia la gobernanza intercultural porque provee los marcos de referencia para la flexibilización de las identidades culturales a través de la consideración de los flujos de movilidad de las personas, bienes, servicios e idea.

Las teorías sobre la gobernanza intercultural se desarrollan y evolucionan a fin de facilitar la implementación de procesos de iniciativas entre los diversos grupos de interés que tienen una diversidad de antecedentes culturales. La teoría de contingencias sostiene la existencia de un patrón de estructuras institucionales y organizacionales que son correctas en cada sociedad. Así las estructuras y formas de la gobernanza intercultural democrática se ejercitan en un determinado contexto cultural.

\section{Noción de gobernanza}

La noción de gobernanza es muy amplia porque trasciende al concepto de gobierno en toda forma organizacional, públicas o privadas, no gubernamentales o de lucro y permite los contactos e interacciones entre los más diversos actores y grupos de interés o stakeholders. La gobernanza se conceptualiza como el conjunto de funciones y acciones de la capacidad institucional del gobierno para gestionar la interculturalidad como política. Como categoría, la gobernanza es el ejercicio de la capacidad del gobierno para relacionar recursos, medios y objetivos institucionales.

El concepto de gobernanza ha trascendido el aparato formal de gobierno en las últimas décadas para incluir los procesos de toma de decisiones y las formas de su implementación (Weiss, 2000). El concepto de gobernanza se ha convertido de una palabra de actualidad de 
uso como gobernanza corporativa, después de que se inició como gobernanza en Francia, luego se extendió su uso a Inglaterra y Estados Unidos (Le Roy 2005).

La noción de gobernanza implica el conjunto de políticas, estrategias, sistemas e instrumentos de gobierno orientados para la formulación y la implementación de procesos de interculturalidad en cualquier ámbito y nivel institucional y organizacional. La noción de gobernanza la conceptualiza como un sistema de valores, políticas e instituciones por los cuales una sociedad gestiona sus asuntos económicos, políticos y sociales a través de la interacción dentro y entre el Estado, la sociedad civil y el sector privado (Dwivedi, 2001). Los sistemas de la gobernanza intercultural se sustentan en políticas públicas y programas democráticos desde una perspectiva de diversidad cultural para la promoción del desarrollo económico, político y social y que tienen como finalidad la consecución de la paz mundial.

El Banco Mundial define la gobernanza como el uso de los recursos institucionales para la gestión de los problemas y asuntos de la sociedad, capacidad del Gobierno para formular e implementar políticas, y el uso de las instituciones y la capacidad de crear un contexto de colaboración para distribuir recursos y coordinar la actividad del Gobierno.

La noción de la gobernanza global desde el enfoque de la antropología legal tiene que separarse de la visión hegemónica occidental si se quiere transformar las realidades considerando sus diferentes implicaciones y consecuencias (Cavanagh \& Mander 2004, de Sousa Santos \& Rodríguez-Garavito, 2005, Kothari 1990). La noción de gobernanza en su connotación administrativas se asocia con la corporación entendida como una modificación de gobierno en un acercamiento legal de la estructura piramidal a la de red (Ost \& van de Kerchove 2002). 


\section{Interculturalidad}

La interculturalidad es un factor del desarrollo humano y de la humanidad en su especie. En la era de crecientes procesos de integración global económica, también se experimentan procesos de integración social, política y de una creciente interculturalidad que queda de manifiesto en el aumento de los intercambios masivos de información, tecnología, personas, bienes y servicios que están modificando nuestros estilos de vida y la coherencia de las sociedades.

El interculturalismo es un concepto holista aplicable a la forma de hacer la política mediante el desarrollo de una visión y compromiso entre los más diversos actores y grupos de interés sociales, económicos y culturales (Zapata Barrero, \& Pinyol Jiménez, 2013). El concepto de interculturalidad busca enfatizar los procesos culturales en los económicos y sociales en sus múltiples arreglos y formas de gobernanza y las actividades económicas formales e informales.

La interculturalidad es un enfoque pertinente para el análisis de los mecanismos a través de los cuales se regulan las relaciones entre los individuos y los grupos de interés en una sociedad determinada. Son los valores la esencia misma de las relaciones de interculturalidad entre los individuos porque motivan la apreciación de otras formas de ser, de actuar y de vivir. Cuando el sistema económico, político y social tiene una amplia capacidad para cubrir las relaciones de interculturalidad y se combina con las interconectividades del mismo sistema, la nueva estructura requiere de una gobernanza intercultural.

Las políticas culturales orientadas a la preservación y promoción de la interculturalidad son alentadas y conducidas a este fin. Por ejemplo, los activos intangibles del patrimonio de los pueblos es una fuente de empoderamiento participación de las comunidades involucradas 
en la preservación de valores, costumbres y tradiciones y por lo tanto requiere de una forma de gobernanza intercultural.

Los derechos y libertades humanas, la cohesión social y la gobernanza se fortalecen con la interculturalidad de la sociedad. La emergencia de un liderazgo intercultural con líderes cívicos y políticos que tienen una diversidad de antecedentes culturales, étnicos, raciales, religiosos, etc., incrementa las posibilidades de ampliar las competencias de interculturalidad, de crear los espacios para el diálogo intercultural.

\section{Gobernanza intercultural}

La noción de gobernanza intercultural no ha sido delimitada y definida claramente, a pesar de que el concepto de interculturalidad está presente en el debate. La gobernanza intercultural y la administración de la diversidad son retos para este siglo XXI, el cual se configura y delimita como el conjunto de nociones, conceptos, principios, políticas y estrategias, instrumentos y métodos, tareas y acciones de interculturalidad que sirven de guía. La interculturalidad de la gobernanza es una condición de facto que tienen las organizaciones y las instituciones de las sociedades para su desarrollo futuro.

La noción de buena gobernanza intercultural formada por el sistema legal, político, social y económico tiene implicaciones que pueden ser percibidas como un concepto vago, si se considera como concepto (Baron 2003, Gaudin 2002, Simoulin 2003). Una mala gobernanza se caracteriza por la arbitrariedad de la toma de decisiones burocráticas en sistemas que son injustos, corruptos e ilegales, donde se cometen abusos de los poderes debido a una sociedad civil que no está involucrada en la esfera pública (World Bank, 1994; Treisman, 2000).

La noción de gobernanza intercultural hace referencia con las demandas relacionadas con la gestión de la diversidad que interpela a la gestión del gobierno local a dar una respuesta 
institucional (Zapata Barrero, 2014). La gobernanza intercultural incorpora las preocupaciones derivadas de la diversidad en las funciones y tareas del gobierno local.

Así, la noción de gobernanza intercultural implica la capacidad de gestión de las políticas interculturales. La gobernanza intercultural implica la noción como el conjunto de instrumentos y dispositivos de gobierno necesarios para poder desarrollar políticas interculturales. El enfoque de la gobernanza intercultural proporciona los instrumentos y mecanismos institucionales y de gobierno para la formulación e implementación de las políticas interculturales en todas las funciones y actividades de los gobiernos locales.

Los mecanismos de gobernanza, los valores y las instituciones juegan un papel relevante en la diversidad y sus implicaciones en los resultados económicos y sociales. Eventos recientes han puesto en crisis ciertas ideas sobre transparencia y rendición de cuentas que requieren una nueva forma de pensar desde la perspectiva de la gobernanza intercultural en las actuales circunstancias. La interculturalidad en la gobernanza se sustenta en los valores de la confianza, transparencia y la rendición de cuentas de la diversidad de interacciones entre los ciudadanos, las instituciones y comunidades, lo cual sirve de fundamento para la gobernanza democrática de la diversidad cultural.

Como una categoría, la gobernanza intercultural establece los objetivos del ejercicio del gobierno como la capacidad para el uso de la diversidad cultural como recursos y medios institucionales para lograr los resultados deseados. La gobernanza intercultural promueve una mejor distribución de los recursos entre los diferentes grupos de interés.

La noción de gobernanza intercultural se establece como el conjunto de acciones de gobierno que facilitan la capacidad institucional para incorporar la lógica del ejercicio de la diversidad cultural en todos los procesos de gestión gubernamental. La gobernanza intercultural es un concepto que relaciona el gobierno en cualquier nivel institucional y organizacional con las políticas, estrategias, planes y programas de gestión estratégica para la interculturalidad. 
El concepto de gobernanza intercultural implica el diseño de políticas y estrategias para el desarrollo y el fortalecimiento de las estructuras de gobernanza y la formación del liderazgo intercultural, el fortalecimiento de programas para el diálogo en la diversidad de los actores involucrados, los procesos de mediación y resolución de conflictos. La gobernanza intercultural incluye una amplia gama de actividades de los actores que involucran a todas las personas y grupos de interés de todas las comunidades culturales, los actores formales e informales, las instituciones políticas y gubernamentales, organizaciones civiles y no gubernamentales, organizaciones voluntarias y filantrópicas, organizaciones privadas y de negocios y todo tipo de estructuras formales e informales de organización (Frederickson, 1997).

Las organizaciones voluntarias que han sido en parte removidas de la esfera política pero no de la esfera pública son las promotoras de interrelaciones y contactos de buena voluntad y confianza, de comportamientos de cooperación y tolerancia, las cuales cimientan las uniones de la sociedad civil y fortalecen la gobernanza intercultural.

Entre las políticas relevantes de gobernanza intercultural están las relacionadas asegurar igualdad de oportunidades mediante el ejercicio pleno de los derechos humanos y con el combate a los prejuicios y discriminaciones, el diseño de estructuras de instituciones de gobernanza del Estado de Derecho con capacidad para desarrollar y fortalecer las competencias de interculturalidad, la participación democrática ciudadana, las relaciones de cooperación y confianza entre las instituciones intergubernamentales, con las organizaciones no gubernamentales y de la sociedad civil, los servidores públicos y en general con todos los grupos de interés involucrados. 
La interculturalidad de la gobernanza alienta los derechos humanos proclamados universalmente que deben estar en pleno ejercicio, fortalece la cohesión social y promueve nuevas formas de gobernanza democrática. La interculturalidad de la gobernanza se convierte en un instrumento para el manejo y el ejercicio pleno de los derechos humanos. La gobernanza intercultural es un proceso institucional y organizacional que contribuye a la apropiación y al ejercicio pleno de los valores de la libertad y los derechos humanos universalmente reconocidos.

La promoción de los derechos humanos como una garantía para fortalecer las relaciones entre las naciones se centra en asuntos relacionados con los procesos de inclusión, confianza, respeto por las creencias religiosas, diversidad cultural, tolerancia por los estilos de vida, etc., en suma en las nuevas formas de la gobernanza intercultural global. La interculturalidad de la gobernanza se ve favorecida por la universalidad de los derechos humanos.

Los derechos humanos otorgan la garantía para cada uno de los individuos su ejercicio pleno a través del reconocimiento de la interculturalidad, la que a su vez refuerza la cohesión social y las nuevas formas de gobernanza. El reconocimiento de la gobernanza intercultural alienta los derechos humanos universalmente reconocidos y garantizados para cada persona en su pleno ejercicio, lo que a su vez refuerza la cohesión social y la renovación de modos y formas de gobernanza democrática.

La proclamación universal de los derechos humanos y los principios de la gobernanza intercultural democrática reafirmados por las instituciones internacionales, se centra en el reconocimiento de las diferencias en la diversidad cultural con el objetivo común de lograr un mejor entendimiento mutuo.

La democratización de los procesos de la gobernanza intercultural y la proclamación de los derechos humanos, son la piedra angular para lograr una humanidad común en la paz universal. 
Muchos de los modelos de gobernanza intercultural adoptados en otras sociedades y que reconocen la unidad en y por la diversidad, que considera la humanidad inherentemente compartida han logrado resultados positivos en prevención de conflicto y la consecución de la paz. En el desarrollo de la interculturalidad de la gobernanza, ésta debe considerarse como totalmente compatible con los fundamentos de la humanidad común y los principios de los derechos humanos y libertades universalmente aceptados.

La dimensión cultural de la participación ciudadana es central a la noción de la gobernanza intercultural, sobre todo como un enfoque para el diseño de las políticas públicas relacionadas con los procesos de los contactos y las relaciones de cooperación y confianza con todos los ciudadanos y los grupos de interés involucrados. Estos procesos de interacción entre los diversos actores se facilitan por las estructuras de gobernanza para el diseño y administración de las políticas públicas de gobernanza intercultural.

La gobernanza intercultural es un mecanismo para gestionar y monitorear las políticas públicas relacionadas con la interculturalidad de la gobernanza para mejorar la implementación de los derechos y libertades universalmente reconocidos. Las políticas públicas deben orientarse a fortalecer la preservación y la promoción de la interculturalidad de la gobernanza.

La cultura es una dimensión estrechamente vinculada con la gobernanza intercultural, que determina el capital social, custodia los valores de los individuos que sustentan las bases para la confianza entre los ciudadanos y las instituciones públicas, y por lo tanto, delimita la esfera pública de acción y sustenta las bases para la gobernanza intercultural. La formación de capital social está relacionada con la fortaleza de la gobernanza intercultural cuando los contactos, las interrelaciones y las interdependencias están estrechamente vinculados en relaciones de cooperación y confianza entre los diversos actores y grupos de interés. 
La gobernanza intercultural implica el diseño de políticas públicas orientadas a ofrecer igualdad de oportunidades para todos los ciudadanos en materia de servicios independientemente de su diversidad cultural. Igual de importante es el diseño de instrumentos y mecanismos de estructuras institucionales para la provisión de los servicios que satisfagan las necesidades de los grupos minoritarios mediante el combate a la discriminación y los prejuicios y en defensa de los derechos humanos, sociales, políticos y económicos.

La interculturalidad de la gobernanza puede proveer los medios para que los grupos minoritarios en una sociedad obtengan la confianza para la renovación de las formas más democráticas mediante el reconocimiento y la valoración de sus contribuciones. El acceso al poder político de los grupos minoritarios, incluyendo a las mujeres, que son además miembros de sus comunidades, es un asunto relevante que tiene relación con el sistema y mecanismos de gobernanza que debe ser respetuosa del contexto local.

Desde la perspectiva estructural, la gobernanza intercultural se refiere a la gestión del conjunto de recursos institucionales y sociales para el diseño e implementación de la estrategia de interculturalidad. Desde la perspectiva de restricciones, la gobernanza intercultural son las limitaciones estructurales, institucionales, económicas y subjetivas que limitan el desarrollo y la implementación de la gobernanza cultural (Zapata Barrero, 2014).

El tema de la gobernanza intercultural preocupa al proyecto Intercultural Cities del Consejo de Europa. El marco conceptual y teórico metodológico de la gobernanza intercultural ha sido propuesto por el programa Ciudades interculturales del Consejo de Europa. Este programa se refiere a la gobernanza y a las políticas para las comunidades que son diversas, aunque la relaciona pobremente con el concepto de gobernanza. El acercamiento a la gobernanza intercultural de Intercultural Cities se sustenta en que el interculturalismo es un concepto holista que configura un conjunto de conceptos, acciones, instrumentos y políticas y no una política específica aplicable a los nuevos retos que resultan de la nueva realidad de administración de la diversidad en el siglo XXI. 
La gobernanza intercultural enfoca las tareas y funciones de la administración local para expandir sus alcances en las interrelaciones y contactos entre las personas y los grupos de interés. El acercamiento a la gobernanza intercultural de Ciudades Interculturales trata de enfrentar al reto gestionar las políticas interculturales configurando conceptos, políticas. Instrumentos y acciones. Los documentos del programa de Intercultural Cities del Consejo de Europa limita la noción de gobernanza intercultural a la gobernanza y las políticas para las comunidades diversas.

La gobernanza intercultural debe adaptarse con otros elementos del desarrollo tales como la participación democrática ciudadana, el fomento y desarrollo de las competencias interculturales a través de programas de formación, la creación de espacios para el ejercicio del diálogo intercultural en todos los niveles de interacciones e interrelaciones. Los procesos de interculturalidad de la gobernanza proveen las formas de interrelación, las prácticas y los estilos de interacción que coexisten en los diferentes grupos de interés en contextos específicos.

La propuesta del Índice de Gobernanza Intercultural (IGI) pretende analizar y reflexionar sobre las políticas de interculturalidad para mejorar los compromisos de los gobiernos locales. El índice de interculturalidad de ciudades determina el grado de movimiento en el acercamiento a la gobernanza intercultural. El Índice de Gobernanza Intercultural (IGI) está compuesto de indicadores mínimos para evaluar las políticas interculturales de gobernanza en las ciudades. 


\section{El diálogo intercultural}

El diálogo intercultural es un proceso en que participan y colaboran todos los grupos de interés involucrados en la agenda para el desarrollo humano, los derechos humanos, una gobernanza democrática que implique mayor inclusión social, justicia y paz. La gobernanza intercultural a cualquier nivel depende de la capacidad para cultivar espacios de diálogo (López Pagán, 2010). La gobernanza intercultural en cualquier nivel institucional y organizacional es materia de abrir y cultivar espacios para el diálogo (Lähdesmäki, 2014). La gobernabilidad democrática de los procesos de implementación de la interculturalidad requiere de una intensa participación democrática ciudadana como la piedra angular sobre la que se fortalecen las competencias y el diálogo intercultural.

En la nueva era de la globalidad social y económica se requiere resolver los retos que representan la diversidad de las sociedades para avanzar en el diálogo intercultural para profundizar el desarrollo de la gobernanza intercultural de los pueblos. Es a través del diálogo intercultural que se incorporan, se apropian, se protegen mejor y se defienden los derechos humanos y las libertades universalmente aceptadas en el contexto cultural mismo de las sociedades, instituciones y organizaciones (An-Na'im, 1992, 2002, 2003; Forsythe and McMahon, 2003). El diálogo intercultural es un instrumento de interacción social que contribuye a la gobernanza de la diversidad de los individuos que forman una sociedad y sus contactos e interrelacione con otras sociedades.

El diálogo intercultural reconoce y favorece la confianza y el respeto mutuo entre los individuos de una sociedad y entre las diversas sociedades. Además, el diálogo intercultural protege y promueve aquellos valores que fortalecen la diversidad de las culturas y que se convierten en los principios de los programas y políticas públicas.

El reconocimiento del diálogo intercultural en la gobernanza intercultural reduce los conflictos y las tensiones de las sociedades monoculturales y multiculturales en las que una 
mayoría monocultural se confronta con el reconocimiento de los derechos de las minorías multiculturales sociales. En sociedades multiculturales se experimentan situaciones en donde los diferentes grupos, el mayoritario y los grupos minoritarios que buscan y luchan por reconocimiento, muestran hasta cierto grado aceptación y tolerancia entre sí pero se presentan conflictos típicos de una sociedad dividida.

La gobernanza democrática de la diversidad cultural ha sido un reto para el desarrollo de las sociedades, incluyendo las que se dicen avanzadas, debido a las imperfecciones que los modelos de multiculturalidad, monoculturalidad, y diversidad cultural han presentado. Más allá del multiculturalismo y de la diversidad cultural, las nuevas formas de gobernanza intercultural está más en contacto y a tono con las prácticas sociales de coexistencia de las personas y grupos de interés de los contextos culturales específicos.

La creación de espacios de diálogo abiertos, ya sea físico o virtual, a la participación de todos, es una condición necesaria para el cultivo de la gobernanza intercultural, en cualquiera de los niveles. Así la gobernanza intercultural tiene que ser desarrollada en cada uno de todos los niveles.

Por ejemplo, a nivel de organización, los ambientes organizacionales que ofrecen una diversidad de culturas corporativas para el diálogo intercultural, son los espacios con sus contextos para estudiar y analizar las formas de gobernanza intercultural a través de la multiplicidad y complejidad de las interrelaciones y contactos de las personas y los grupos de interés. La gobernanza intercultural se integra en la gobernanza corporativa al interior del funcionamiento de los negocios y a informar el desarrollo e implementación de las estrategias de negocios. Este enfoque de gobernanza corporativa intercultural tiende a ser más multinacional y diversificado a fin de considerar el involucramiento de un amplio rango de importantes colaboradores y grupos de interés. 
El diálogo intercultural es una herramienta y una tarea que requiere de varias acciones. Políticas, iniciativas y programas en gobernanza intercultural deben privilegiar el diálogo intercultural, alentar la participación democrática intercultural orientada a reforzar las capacidades de las instituciones que contribuyen al desarrollo de la gobernanza. Algunas de las iniciativas y programas prioritarios que pueden efectuar los gobiernos a través de las relaciones intergubernamentales para implementar y acrecentar la gobernanza intercultural, son entre otros, en materia de derechos humanos, educación y formación global, gobernabilidad democrática, etc.

Una tarea importante es la determinación de los requerimientos para el desarrollo de instrumentos, mecanismos y herramientas institucionales y organizacionales que tengan como finalidad el diseño e implementación de políticas y estrategias de gobernanza intercultural.

La promoción del diálogo intercultural como condición para crear el ambiente que da sustento a la gobernanza intercultural, fomenta valores de tolerancia, comprensión y entendimiento entre los individuos con diferencias y diversidades de antecedentes étnicos, religiosos, culturales, etc. y genera relaciones de cooperación y confianza. La religión es una dimensión relevante de los procesos de gobernanza intercultural delas sociedades y una fuerza generadora y motivadora de valores éticos, culturales, económicos, políticos, etc.

Es por lo tanto, el ambiente del diálogo intercultural, el cemento que une y mantiene a los individuos de una sociedad juntos. Además, este ambiente constituye el medio efectivo para desarrollar la gobernanza intercultural que construye estructuras sociales más participativas, más igualitarias, más democráticas, más inclusivas y crea espacios más dinámicos para propiciar el desarrollo humano de todos los ciudadanos.

Para fortalecer el diálogo intercultural en las organizaciones se requiere el desarrollo de una cultura política que derive en la formulación de una política pública para alcanzar la gobernanza democrática de la diversidad cultural, capaz de reconocer y aceptar los derechos, la participación y la cooperación de todos los grupos de interés involucrados pero especialmente de los minoritarios. Bajo el principio de que la dimensión cultural de la política pública es esencial para los procesos de gobernabilidad intercultural a través de la creación 
de contactos e interrelaciones entre los sectores de la sociedad civil, el gobierno y los negocios.

El desarrollo de una cultura política que valora la naturaleza de la interculturalidad de la gobernanza como un conjunto de derechos humanos, sociales, políticos y económicos, y los principios y valores democráticos del ejercicio de las libertades fundamentales, el Estado de Derecho, la no discriminación en función de origen étnico-racial, religioso, etc., la tolerancia, la pluralidad, las relaciones de cooperación y confianza, el respeto mutuo, etc. Entre otras características de la gobernanza intercultural se pueden mencionar la autoridad pública imparcial delimitada por el Estado de Derecho.

Para lograr esto, es necesario establecer los nuevos mecanismos a través de políticas de cooperación que incidan en el fortalecimiento de la confianza y el entendimiento de los valores de la diversidad.

El diseño e implementación de las estructuras de gobernanza adecuadas en las organizaciones de la comunidad y en las instituciones públicas del gobierno local, es una de las condiciones para facilitar el diálogo intercultural a nivel más bajo. El diálogo intercultural requiere de espacios que deben estar abiertos para todas las personas y los grupos de interés. Además, se deben ser creados los espacios para el diálogo de la gobernanza intercultural en todos los niveles de gobierno. La configuración de espacios públicos para el diálogo abiertos a todos, debe optimizar dinámicas y ritos existentes. 


\section{Participación ciudadana}

Con los cambios económicos y políticos a escala global en la década de los ochentas, se considera el hecho de que una gobernanza intercultural es factible porque emerge espontáneamente con la participación de grupos de interés en asuntos que les son comunes. La estrategia global para promover la buena gobernanza intercultural es el instrumento ideal para prevenir, resolver, dirimir y abatir conflictos y construir las instituciones que establezcan la justicia, la igualdad, la equidad, la libertad y la construcción de la paz.

La gobernanza democrática de la interculturalidad permite una mayor participación ciudadana democrática y la creación de espacios de diálogo intercultural donde se ejercitan y refuerzan la adquisición de competencias interculturales con la finalidad de que los humanos puedan vivir juntos, en iguales términos de dignidad, libertad, justicia y paz. La gobernanza intercultural se preocupa por la participación y representación democrática entre una mayoría y las minorías, el uso de las competencias y facultades interculturales en los asuntos de gobierno para gestionar las iniciativas que incorporen la diversidad cultural, y todavía más allá, la interculturalidad.

La ciudadanía participativa democrática y active encuentra muchos medios que le ayudan a promoverse para servir a los mecanismos de gobernanza intercultural en forma más adaptado a la diversidad de las personas en una sociedad. Un nivel fuerte de capital social y de confianza social y la participación de la ciudadanía y las organizaciones no gubernamentales y de la sociedad civil se relacionan en un sistema de gobernanza democrática intercultural.

La confianza mutua es el punto de partida de la formación de capital social que ayuda a forjar mayor cohesión social y un mayor consenso entre las personas en cualquier nivel de gobierno. La confianza mutua es un acercamiento universalista que sustenta y fortalece la coexistencia 
pacífica de las sociedades. El ejercicio pleno de los derechos humanos y las libertades fortalecen la cohesión social que faculta la gobernanza intercultural democrática.

Con el sustento de los derechos y las libertades humanas, las relaciones de cooperación y confianza entre los individuos y la formación de la cohesión social que inciden en la interculturalidad de la gobernanza, convergen en una creación de relaciones de coexistencia pacífica y de paz en cualquier estructura social. La gobernanza intercultural democrática facilita la cohesión social en sociedades que tienen una diversidad cultural y sociedades multiculturales. Por otra parte, la gobernanza intercultural es a su vez un instrumento que contribuye a fortalecer la cohesión social a través del diseño y desarrollo de estructuras institucionales y organizacionales que faciliten las nuevas formas de participación ciudadana en la gobernanza intercultural.

Los programas de gobernanza intercultural en los gobiernos locales intentan gestionar estratégicamente los procesos de interculturalidad de la diversidad que se expresa en los diferentes grupos de población y su participación ciudadana, la representación de las minorías en las estructuras y procedimientos de gobierno como el caso de los diferentes grupos de migrantes, la mentalidad y actitudes interculturales de gobernantes, funcionarios electos, empleados y gobernados, etc.

Los gobiernos locales que sustentan las acciones de la gobernanza intercultural a través de sus estructuras institucionales, aseguran la provisión de servicios para satisfacer la diversidad de necesidades de la población. Esta función la realizan a través de la implementación de políticas de igualdad de oportunidades y combate al prejuicio y la discriminación sin comprometer los principios de derechos humanos, democracia y el Estado de Derecho (Zapata Barrero \& Pinyol Jiménez, 2013). Una propuesta para diseñar e implementar las estructuras de gobernanza intercultural en las instituciones de los gobiernos locales es la 
creación de un consejo con facultades para revisar y supervisar las políticas y regulaciones en materia de relaciones interculturales entre ciudadanos y gobierno local.

La participación democrática bajo el esquema de la gobernanza intercultural se puede convertir en una ideología hegemónica que propone un modelo de desarrollo basado en el buen gobierno. Por buen gobierno se ha entendido como la organización para vivir juntos en los que cada quien puede participar. Este concepto va más allá del acercamiento centrado en el Estado nación. Considerando este supuesto, los sistemas políticos y de gobernanza intercultural de los pueblos indígenas se debilitan fuertemente frente a los embates del Estado Nación con su modelo de gobernanza democrático occidental.

El movimiento del acercamiento del gobierno al de gobernanza, representa un desplazamiento del centralismo legalista a acercamientos más plurales de la ley, de tal forma que en el modelo de gobernanza intercultural, el Estado nación es acotado en su estructura jerárquica, piramidal (Griffith, 1986). Este esquema de gobernanza intercultural se despliega de lo particular a lo universal, de lo local a lo global. Sin embargo, el esquema de buena gobernanza parece que no se sustenta y que existe un vacío legal-político y social porque pivota en una democracia centrada en el Estado, en los derechos humanos y el desarrollo.

La gobernanza intercultural se sustenta en nuevas formas de participación ciudadana basada en valores compartidos que contribuyen a fortalecer la cohesión social a través del empoderamiento de la diversidad cultural de las personas y los grupos de interés. La diversidad del patrimonio intangible proporciona ejemplos de modos y formas de gobernanza democrática centrada en el empoderamiento y una mayor participación de ciudadanos de todas las comunidades.

Este empoderamiento les permite a las personas tener mayores elecciones más allá de confinarlos a tribus y clanes monoculturales sino que alentando en ellos las posibilidades de 
apertura a nuevas y diferentes culturas de otros. Entre estas políticas culturales que apoyan la interculturalidad de la gobernanza incorporan en su agenda la necesidad de encontrar medios para extender la participación ciudadana para aquellos que están en las marginalidades de la sociedad (Council of Europe, 1997).

El uso de instrumentos de gobernanza intercultural participativa permite empoderar a los marginados de una sociedad y a los grupos minoritarios que permanecen en las marginalidades, sobre todo a aquellos que no han tenido las oportunidades de acceso a la educación. Cuando estas responsabilidades se realizan se está en posibilidades de efectuar el diálogo intercultural en donde bajo el principio de subsidiariedad se alienta el empoderamiento de los ciudadanos y se reconoce la importancia que tiene la gobernanza intercultural. La gobernanza intercultural está más centrada en el desarrollo de los derechos y las libertades humanas que van más allá de los enfoques de diversidad cultural, multiculturalismo e iniciativas de empoderamiento (UNESCO, 2003).

\section{Desarrollo y formación de competencias de gobernanza intercultural}

Un aspecto importante del diálogo intercultural son las inversiones en el desarrollo y formación de competencias interculturales y de diversidad cultural que hacen los diferentes grupos de interés, a través de procesos de reconciliación y cooperación. Estas inversiones contribuyen a realizar nuevos acercamientos al desarrollo humano sustentable que facilita el ejercicio pleno de las libertades humanas y los derechos humanos que son reconocidos universalmente.

Las competencias de gobernanza intercultural deben ser desarrolladas a través de programas de formación. Una vez que se conoce la situación real después del diagnóstico realizado con los indicadores de gobernanza intercultural, lo importante es tener las facultades y las competencias para intervenir los procesos de gobernanza intercultural. Los indicadores de la 
gobernanza intercultural proporcionan una radiografía sobre la situación actual de la implementación de políticas interculturales, que permite analizar, determinar causas y consecuencias de su gobernanza con el propósito de mejorarla. El tener indicadores ayuda mucho para analizar la situación real contra la situación ideal deseada.

La conceptualización de tipologías de indicadores puede contribuir a avanzar el debate sobre la gobernanza intercultural.

Desde la perspectiva de la gobernanza intercultural se promueven nuevos arreglos estructurales institucionales que faciliten los procesos de cooperación y consulta con todos los grupos de interés a niveles locales, formación de competencias centradas en la interculturalidad, delegación de responsabilidades, promoción de fondos para solución de problemas derivados de la interculturalidad, etc. Los esfuerzos intergubernamentales a cualquier nivel deben centrarse en la promoción del aprendizaje y entendimiento de las competencias interculturales para el diálogo político intercultural en asuntos tan delicados como los relacionados con la migración. La diversidad cultural de la inmigración necesariamente requiere de un tratamiento de gobernanza intercultural dadas las implicaciones con las dinámicas poblacionales.

La educación tiene una función relevante y juega un rol instrumental para diseñar y estructurar las nuevas formas de gobernanza intercultural que facilite a las personas lograr su propio desarrollo económico, social, político y cultural. Este desarrollo redundará en el logro de una fuerte cohesión social. El contenido local de los planes y programas de estudios en educación deben concentrase más en el desarrollo de las habilidades interculturales.

\section{Niveles de gobernanza intercultural}

Los espacios públicos de diálogo son esenciales para la gobernanza intercultural en cualquiera de los niveles institucionales y organizacionales en los que se manifiesta (López 
Pagán, 2010). En los diferentes niveles en que se manifiesta la gobernanza intercultural simplemente es materia del desarrollo en los espacios correspondientes tales como en los gobiernos locales, mercado, organizaciones sociales y civiles, iglesias, universidades, centros culturales y recreativos, etc. (Lähdesmäki, 2014).

La gobernanza intercultural se modifica desde la base misma de las interacciones y contactos de las personas quienes participan más directamente en la conducción de sus propias vidas, porque les permite participar en las agendas e iniciativas establecidas por los gobiernos locales, aunque no necesariamente participen en la formación de las agendas (Campbell 1997, Parthasarahty 2005, Rahnema 1997, 2003).

La gobernanza intercultural muestra características de interactividad holista en estructura de redes que posibilita la toma de decisiones democráticas y las elecciones políticas, sino que también evacúa las diferencias del poder entre los diversos actores y grupos de intereses de un nivel de sistema legal, económico, político y social. Este acercamiento de gobernanza posibilita que los actores involucrados en las diferentes redes se estructuren en campos sociales semi-autónomos (Moore 1973, 1983). La interculturalidad de la gobernanza debe estar basada en las dimensiones culturales de los aspectos económicos, políticos y sociales del nivel de unidad.

Las políticas culturales de los diferentes niveles de gobierno que están orientadas a empoderar a las personas y grupos de interés que están en desventaja, son relevantes para el diseño e implementación de políticas públicas que dan sustento a la formación de comunidades que son capaces de crear estructuras y formas de gobernanza intercultural. Por lo tanto, la cultura del nivel organizacional o de gobierno debe considerarse como un factor relevante, quizás el más importante, de la gobernabilidad intercultural y para la prevención y resolución de conflictos. 
En los diferentes niveles de gobernanza intercultural, desde los organizacionales, gobiernos locales, regionales, nacionales, internacionales y globales, las dimensiones del diálogo intercultural implican la participación de los diferentes grupos de interés a stakeholders y de la ciudadanía democrática, la adquisición y desarrollo de las competencias interculturales y la construcción de un espacio para ejercitar diariamente el diálogo intercultural. Es necesario un enfoque de gobernanza intercultural para la identificación de las buenas prácticas en todos los niveles institucionales y organizacionales de gobierno, de la sociedad civil, no gubernamentales, de negocios, etc.

La gobernanza intercultural involucra a todos los grupos de interés de cualquier nivel de organización para participar y contribuirá los procesos de diseño e implementación de las políticas. Por ejemplo, a nivel de gobierno local, los grupos de interés involucrados en las responsabilidades que implica la gobernanza intercultural con la participación democrática y cooperación de las propias autoridades locales y regionales, los miembros de los diferentes órganos y organismos de gobierno, las instituciones académicas y de investigación, las instituciones de cultura y medios de comunicación, las organizaciones no gubernamentales, profesionales y artistas de las industrias culturales, etc.

Un elemento crítico para la implementación de los procesos de la gobernanza intercultural en las instituciones públicas y en las organizaciones sociales o privadas, es la determinación de lo que es necesario cambiar para lograr los objetivos estratégicos planteados por lo más altos niveles directivos que defienden o que promueven este enfoque. Las personas y los grupos de interés participan con sus propias expresiones de interculturalidad para buscar su propia realización de objetivos comunes con el sustento de una forma de gobernanza. De esta forma, la respuesta es el desarrollo de la gobernanza intercultural que tiene serias implicaciones para los mecanismos de compartir el poder y el aseguramiento de los derechos y libertades de los individuos. 
Los acercamientos a la gobernanza intercultural varían considerablemente en algunos principios y temas, dependiendo de las situaciones específicas. Entre estos temas se pueden destacar los niveles de promoción de la libertad de expresión e información, el desarrollo de programas de cooperación intergubernamental, intersectorial, en la gobernabilidad democrática en todos los niveles, etc.

Los procesos de gobernanza intercultural en las organizaciones a cualquier nivel alientan la toma de decisiones y apoyan la emergencia de liderazgos civiles y políticos provenientes de diferentes antecedentes que se convierten en culturalmente competentes y conocedores (Zapata Barrero \& Pinyol Jiménez, 2013). El establecimiento de los procesos de gobernanza intercultural alientan los procesos de toma de decisiones en las estructuras institucionales del sector público y en las organizaciones de la sociedad civil y no gubernamentales.

La gobernanza intercultural democrática establece modos, estructuras y formas de toma de decisiones de gobierno a partir de consideraciones de la composición de la diversidad cultural y multicultural de las personas y los grupos de interés en las sociedades que muestran una gama amplia de valores, creencias, patrones de comportamiento de estilos de vida, formas de ser y de pensar, costumbres, mitos, etc.

La gobernanza intercultural profundiza la implementación de las políticas y estrategias interculturales en los niveles de gestión local. La gestión local encuentra que sus límites para el desarrollo de sus actividades pueden ser rebasados por un enfoque de gobernanza intercultural. En algunas ciudades se desarrollan programas de bienvenida a los migrantes con acciones que tratan sobre algunos aspectos específicos de la diversidad intercultural tales como la remoción de restricciones institucionales. Estas acciones implican el involucramiento de diferentes niveles de gobierno para trabajar en una forma más coordinada para mejorar la gobernanza en materia de políticas interculturales. 
El programa de ciudades en Europa intenta revisar las políticas y estrategias de los servicios ofrecidos por las instituciones de los gobiernos locales para crear las adecuadas estructuras y mecanismos que faciliten los procesos de integración de los grupos minoritarios y de migrantes a la normalidad social, política, cultural y económica. Algunos de estos instrumentos y mecanismos que facilitan los procesos de gobernanza intercultural son la visión y estrategia de la ciudad intercultural, los modelos de gobernanza para el desarrollo y en índice de interculturalidad.

Deben ser las propias comunidades locales las que gestionen sus propios procesos de gobernanza intercultural acorde con sus propios niveles de desarrollo a través del acceso apropiado a sus propios recursos, facilidades y programas de desarrollo y formación de capacidades interculturales. La Tanda es una organización no gubernamental no profesional que a nivel de comunidad formada por residentes, aplica la lógica de la gobernanza intercultural. De acuerdo al reporte de Palomera y Aramburu (2012), los miembros de esta organización a ras de tierra que son migrantes sin formación técnica, participan en la política local con la finalidad de mejorar sus condiciones de vida y que se afectan directamente por sus conflictos internos, situación que contribuye a considerar los puntos de vista divergentes de los diferentes actores.

\section{Gestión de la gobernanza intercultural}

La capacidad de gestión de la gobernanza intercultural tiene como finalidad el diseño e instrumentación de la política intercultural para el logro de objetivos para la promoción de la diversidad en términos de la estabilidad, la cohesión y el desarrollo de las capacidades de su población (Zapata-Barrero, 2013). La gobernanza intercultural profundiza las políticas públicas de interculturalidad a pesar de las restricciones que tiene la administración local que rebasa la gestión de los asuntos de interculturalidad. 
La gestión de la gobernanza intercultural tiene como dimensiones la estructural y la restrictiva. En la dimensión estructural la gobernanza intercultural implica el conjunto de recursos y medios institucionales y sociales para la gestión de la estrategia de interculturalidad del gobierno local. Esta capacidad para planear, diseñar e implementar la política y estrategia de interculturalidad incluye la instrumentación de los canales y medios de comunicación, el empleo de las redes de los actores sociales y otro tipo de acciones tales como las de formación. No obstante, el ejercicio de la gobernanza intercultural se encuentra limitada por factores subjetivos, económicos, estructurales e institucionales Zapata Barrero, 2014).

La gobernanza intercultural se relaciona con la gestión institucional de la diversidad cultural para dar respuestas a las demandas de una población diversa. En este sentido, la gobernanza intercultural es la capacidad de gestión de las políticas y estrategias para alcanzar las ventajas de la diversidad mediante la obtención de objetivos de política intercultural, en términos de estabilidad, cohesión y desarrollo de las capacidades de la población (Zapata-Barrero, 2013).

La gobernanza intercultural implementa procedimientos, estructuras y comportamientos organizacionales que facilitan el diseño y la incorporación a la gestión pública local de políticas públicas, mecanismos, instrumentos para la implementación de políticas y estrategias de interculturalidad. El modelo de gobernanza intercultural desarrollado y promovido por Intercultural Cities ha sido validado y probado en la gestión pública local. El interculturalismo es una forma de desarrollar una visión para hacer política involucrando a los actores de la administración y a los diversos grupos de interés de la gestión pública local (Zapata Barrero \& Pinyol Jiménez, 2013). Sin embargo, en la perspectiva del modelo hegemónico de gobernanza intercultural lo que no se cuestiona es la supremacía de quienes implícitamente crean e imponen las reglas del juego. 
La gobernanza intercultural es una agenda relevante en el sector privado empresarial y en las agendas de los tomadores de decisiones de la administración y gestión de las organizaciones de gobierno, que implica las operaciones corporativas y las prácticas del gobierno.

\section{Tendencias y retos de la gobernanza intercultural}

La gobernanza intercultural presenta varios retos debido fundamentalmente al ejercicio de la diversidad cultural para la transformación en un principio de coexistencia armónica a través de la promoción de competencias interculturales para una sociedad de paz. Los retos que plantea la gobernanza intercultural priorizan la urgencia de diseñar estructuras, planear rutinas y procedimientos y ejecutar acciones que permitan la implementación de políticas y estrategias interculturales.

Hay una tendencia clara de que el desarrollo de los procesos de interculturalidad contribuyen a revivirlas formas y modos de gobernanza local con la iniciación de nuevas formas de involucramiento público de las personas que son más respetuosas y tolerantes de los valores y puntos de vista de las demás personas (Therrien, 2007. Tierney, 2008). La creación de nuevas formas de gobernanza intercultural debe estar diseñada para dar mayores facilidades para abrazar las diferencias y diversidades culturales de las personas y grupos de interés. De hecho, la gobernanza intercultural representa uno de los principales retos de la diversidad cultural.

El marco de referencia legal también necesita avanzar desde la teoría legal clásica a una teoría más intercultural del derecho, para dar mayor sustento a los retos de las nuevas realidades impuestas por una nueva gobernanza intercultural global. Sin embargo, deben tenerse cuidado de que esta nueva tendencia de gobernanza global se convierta en la ideología hegemónica que trata de imponer una agenda depredadora del desarrollo. Las sociedades, 
instituciones y organizaciones regidas por formas y modos de gobernanza intercultural difícilmente aceptan el dominio externo o interno.

La gobernanza intercultural reconoce las diferencias culturales que son específicas a los diferentes estilos de vida de los ciudadanos, las diversas formas y estructuras institucionales y organizacionales con diferentes modos de producción, lo cual hacen posible la realización de una agenda de desarrollo sustentable aplicable a las diferencias culturales y religiosas (Hasan, 2006). El desarrollo sustentable y la gobernanza intercultural son dos conceptos que marcan tendencias en el desarrollo de las organizaciones, las instituciones y los pueblos para dar respuesta a las nuevas realidades. Estas nuevas realidades que rebasan los límites y alcances del estado nacional se centran en las dinámicas de una mayor participación de la sociedad civil y un modelo de cooperación y corregulación interinstitucional.

Uno de los retos de la gobernanza intercultural es la institucionalización de la misma gobernanza, de tal forma que sea factible determinar los grados de efectividad y eficiencia de las políticas públicas y sus intervenciones. La función de la identidad cultural de los pueblos y comunidades al igual que de las organizaciones e instituciones contribuye al diálogo intercultural con la promoción de modelos de gobernanza intercultural y auto gobernanza democrática que alientan y promueven el desarrollo sustentable. En este sentido, se puede afirmar que los mecanismos de gestión del patrimonio intangible de los pueblos entran en el servicio de la gobernanza democrática.

Las nuevas realidades sociales deben ser tomadas en cuenta bajo las perspectivas del desarrollo sustentable y la gobernanza intercultural desde los retos de la interculturalidad para refundar el Estado de Derecho. A esta nueva tendencia se le denomina la buena gobernanza que enfatiza el diálogo intercultural. Se plantea el cuestionamiento para el diseño y desarrollo del mejor modelo de gobernanza intercultural a partir de los propios recursos interculturales que las mismas sociedades tienen en su stock de instituciones sociales, 
políticas, culturales y económicas, capaz de propiciar y acelerar su propio desarrollo. Uno de los retos de la gobernanza intercultural es la institucionalización de la misma gobernanza, de tal forma que sea factible determinar los grados de efectividad y eficiencia de las políticas públicas y sus intervenciones.

Entre las competencias de la gestión organizacional que tienen una mayor importancia para enfrentar los retos de la gobernanza intercultural en los procesos de globalización económica, incluyen las habilidades para operar en una diversidad de ambientes culturales que se hacen presentes en los contextos organizacionales. Esta tendencia hacia la gobernanza intercultural de las organizaciones e instituciones en sus diferentes niveles, ha hecho necesario un amplio rango de políticas, estrategias, métodos y sistemas sensibles a la interculturalidad de las relaciones y contactos entre las personas de los diferentes grupos de interés.

Para la implementación de formas de gobernanza intercultural de modo más inclusiva para todas las personas, la gestión de la interculturalidad representa retos sociales que implican el establecimiento de formas de gobierno más democráticas que propicien la apropiación y el ejercicio de los derechos humanos y libertades universalmente aceptados. Esta orientación ha hecho necesario que se definan, delineen y diseñen nuevos patrones de gobernanza corporativa intercultural con capacidades para relacionar las diferentes culturas corporativas, sobretodo de aquellas instituciones y corporaciones que operan en diferentes ambientes nacionales.

El más grande de los retos de la gobernanza intercultural es la administración de la diversidad en los programas e iniciativas de gobierno. Las formas de gobernanza intercultural se renuevan con las lecciones aprendidas de otros modelos que han sido adaptados en otras culturas y que reconocen la diversidad cultural más allá del multiculturalismo. Más allá del multiculturalismo y de la diversidad cultural, las nuevas formas de gobernanza intercultural está más en contacto y a tono con las prácticas sociales de coexistencia de las personas y grupos de interés de los contextos culturales específicos. 


\section{Referencias}

An-Na'im, A. A. Toward a cross-cultural approach to defining international standards of human rights: The meaning of cruel, inhuman, or degrading treatment or punishment. A. A. An-Na'im (ed.), (1992). Human Rights in Cross-cultural Perspectives. Philadelphia, Pa., University of Pennsylvania Press.

An-Na'im, A. A. Human Rights Under African Constitutions: Realizing the Promise for Ourselves. Philadelphia, Pa., University of Pennsylvania Press. (ed.). 2003.

An-Na'im, A. A. Cultural Transformation and Human Rights in Africa. London, Zed Books. (ed.). 2002.

An-Na'im, A. A. Human Rights in Cross-Cultural Perspective: A Quest for Consensus. Philadelphia, Pa., University of Pennsylvania Press. (ed.). 1992.

Baron C. La gouvernance: débats autour d'un concept polysémique, Droit et Société, $n^{\circ} 54$, p 329-351(2003).

Campbell B. Reconceptualisation de l'État au Sud - Participation démocratique ou managérialisme populiste », in CRÉPEAU François, Mondialisation des échanges et fonctions de l'État, Bruxelles, Bruylant, 294 p (163-231). (1997).

Cavanagh J. \& Mander JAlternatvies to Economic Globalization. A Better World is Possible, Second Enlarged Edition, San Francisco, Berret-Koehler Publishers, 408 p. ., (2004).

Council of Europe In From the Margins: A Contribution to the Debate on Culture and Development in Europe. Strasbourg: Council of Europe. (1997). http://www.coe. int/t/dg4/cultureheritage/Source/Resources/Publications/Culture/InFromTheMargin s_Short_EN.pdf.

Dwivedi, O. P. The challenge of cultural diversity for good governance. Paper presented at the United Nations Expert Group Meeting on Managing Diversity in the Civil Service, New York, 3-4 May. 2001. http:// unpan1.un.org/intradoc/groups/public/documents/UN/UNPAN000573. pdf

Forsythe, D. P. and McMahon, P. C. Diversity and Human Rights: Area Studies Revisited. Lincoln, Neb., University of Nebraska Press. (Eds.). 2003.

Frederickson, H. G. The Spirit of Public Administration. San Francisco, Calif., Jossey-Bass. 1997. 
Gaudin J-P. Pourquoi la gouvernance? France, Presses de Sciences Po, 137 P. (2002).

Griffiths J., What is Legal Pluralism? , Journal of Legal Pluralism and Unofficial Law, number 24, p 1-55. (1986).

Hasan, Z. Sustainable development from an Islamic Perspective: meaning implications and policy concerns. MPRA Paper No. 2784. 2006. http://mpra.ub.unimuenchen.de/2784/1/MPRA_paper_2784.pdf

Kothari R. Rethinking Development. In search of Humane Alternatives, India, Aspect Publications Ltd, 220 p. (1990).

Lähdesmäki, T. The role of space in the politics of intercultural dialogue. Cultural Encounters The Mosaic of Urban Identities, 28. (2014).

Le Roy É., Pour une anthropologie de la juridicité, Cahiers d'Anthropologie du Droit, $n^{\circ}$ 2004, p 241-247, (2004).

López Pagán, J. La alianza de civilizaciones: una agenda internacional innovadora. Documento de debate. Fundación Ideas. (2010).

Moore S. F., Law as Process - An Anthropological Approach, Great Britain, Routledge \& Kegan Paul, 263 p, (1983).

Moore S. F., Law and Social Change: The Semi-Autonomous Social Field as an Appropriate Subject of Study », Law and Society Review, Summer, p 719-746, (1973).

Ost F., van de Kerchove M., De la pyramide au réseau? Pour une théorie dialectique du droit, Bruxelles, Facultés Universitaires Saint Louis, 596 p. (2002),

Palomera, J., \& Aramburu, M. From the Intercultural Model to its Actual Implementation in a Spanish Neighborhood. (2012).

Parthasarathy D. Taking Participation Seriously. A Critique of Good Governance. Eberhard Ch. (éd.), Droit, gouvernance et développement durable, numéro spécial des Cahiers d'Anthropologie du Droit, Paris, Karthala, 2005, 376 p (307-321), (2005).

Rahnema M. Participation, SACHS Wolfgang (éd.), The Development Dictionary. A Guide to Knowledge as Power, Great Britain, Zed Books, 306 p (116-131), (1997).

Rahnema M., Quand la misère chasse la pauvreté, France, Fayard/Actes Sud, 321 p, (2003),

Simoulin V., La gouvernance et l'action publique: le succès d'une forme simmélienne. Droit et Société, $n^{\circ}$ 54, p 307-328,(2003). 
Therrien, M. Democracy and recognition: building research partnerships. Diogenes, Vol. 55, No. 4, pp. 134-36. 2007.

Tierney, S. Multiculturalism and the Canadian Constitution. Vancouver, B.C.: University of British Columbia Press. (ed.). 2008.

Treisman, D. The causes of corruption: a cross-national study. Journal of Public Economics, Vol. 76, pp. 399-457. 2000.

http://www.sscnet.ucla.edu/polisci/faculty/treisman/causes.pdf

UNESCO .Unesco World Report. Investing in cultural diversity and intercultural dialogue. Unesco Publishing. Published in 2009 by the United Nations Educational, Cultural and Scientific Organization. (2009).

UNESCO. Atelier sur la culture et le développement dans le programme d'action du NEPAD. Paris, UNESCO. 2003 http://ocpa.irmo.hr/resources/docs/NEPAD_Draft_Final_Report-fr.pdf

Weiss, T. G. Governance, good governance and global governance: Conceptual and actual challenges, Third World Quarterly, 21:5, 795-814, (2000).

World Bank. Governance: The World Bank's Experience. Washington, DC, World Bank. 1994

Zapata Barrero, R. Índice de gobernanza intercultural: Informe. GRITIMUPF Policy Series, No. 3. June 2014. Grup de Recerca Interdisciplinari sobre Immigración Departament de Ciències Polítiques i Socials Universitat Pompeu Fabra. (2014).

Zapata-Barrero, R. Diversity management in Spain, Manchester: Manchester University Press. (2013).

Zapata Barrero, R. (2013). The three strands of intercultural policies: a comprehensive view. A critical review of Bouchard and Cantle recent books on interculturalism. Working group series Number 17, Summer 2013. Grup de Recerca. Interdisciplinari en Immigració - UPF. Departament de Ciències Polítiques i Socials Universitat Pompeu Fabra.

Zapata Barrero, R., \& Pinyol Jiménez, G. (2013). Handbook for the design of intercultural policies: summary of the original Spanish version. 